\title{
A Machine Learning Technique to Analyze Surface EMG Signals in Normal and Diabetic Subjects
}

\author{
${ }^{1}$ Anjaneya L.H., ${ }^{2}$ Mallikarjun S. Holi and ${ }^{3}$ Chandrashekar S. \\ ${ }^{1}$ Bapuji Institute of Engineering \& Technology, Dept. of Biomedical Engineering, India. \\ ${ }^{2}$ UBDT College of Engineering, Dept. of Electronics and Instrumentation Engineering, India \\ 3.J.M.Medical College, Dept. of Emergency Medicine, India. \\ anjudvg@yahoo.com; msholi@yahoo.com;drscandru@yahoo.com;
}

\begin{abstract}
The diabetes mellitus results in many musculoskeletal complications and detection of these problems is important in treatment, prevention of disability and improving the quality of life. Many attempts have been made to effectively acquire and analyze the surface electromyogram (sEMG) signals to understand the musculoskeletal problems in diabetes. The development of methodologies to extract the effective features from SEMG still remains a primary challenge. Previous studies have demonstrated that the sEMG signals have nonlinear characteristics. In this study, by combining the nonlinear time-series analysis and the time-frequency domain methods, we propose the wavelet based method to extract the effective features of sEMG signals. The SEMG signals were first acquired and analyzed by the wavelet transform and the features were obtained. Then, these features were used as the input vectors to artificial neural network (ANN) classifier to discriminate diabetic or non-diabetic subject. The results show that significance of proposed feature extraction method with classification of diabetic and nondiabetic subjects with an accuracy of $97.06 \%$.This indicates that the proposed approach can provide important insight into the nonlinear characteristics and the time-frequency domain features of $S E M G$ signals and is suitable for classifying the normal subjects from diabetic subjects. By comparing with other existing methods, the proposed method exhibited more robustness and higher classification accuracy.
\end{abstract}

Keywords: surface electromyography; wavelet; artificial neural network; classification

\section{Introduction}

Healthcare and biomedical frameworks generally requires high level of coordination between health and medical equipment. Maintaining the consistency for biomedical analysis is a crucial task for the researchers and the biomedical experts [1].Some chronic diseases require continuous medical care and patient's self-management to prevent and reduce the complications. Diabetes is one of the most crucial chronic diseases. Diabetes is a gathering of metabolic sicknesses portrayed by hyperglycemia coming about because of deformities in insulin emission, insulin activity, or both. The interminable hyperglycemia of diabetes is connected with long haul harm, brokenness, and disappointment of distinctive organs, particularly the veins, kidneys, nerves, heart, and eyes [2].Diabetes can bring about changes in the musculoskeletal framework, which is the term for muscles, bones, joints, I ligaments, and 
tendons. These progressions can bring about various conditions that may influence your fingers, hands, wrists, shoulders, neck, spine, or feet. Side effects of diabetes related musculoskeletal issues incorporate muscle agony, joint torment or solidness, decreased capacity to move your joints, joint swelling, deformations, and a "pins and needles" sensation in the arms or legs. Some musculoskeletal issues are special to diabetes. The shoulder joint may additionally be influenced in diabetes. What's more, obviously, the feet are vulnerable to issues brought on by diabetes.

Incessant hyperglycemia in individuals with diabetes builds the danger of microvascular harm, which prompts retinopathy, nephropathy, and neuropathy [3]. Diabetic individuals are presented to a hoisted danger of macro vascular inconveniences, where they are two to four times more prone to get cardiovascular diseases (CVD) than individuals without diabetes. Because of these entanglements, diabetes is observed to be the fourth driving reason for worldwide passing by illness [4].Diabetes is the most common cause for neuropathy. Muscles are the vital organ responsible for the movement of the body. Electromyography action is recorded from contracted arm endless supply of the guideline from the mind. Electromyogram (EMG) is utilized to provide the information about the contraction and relaxation of individual muscles during locomotion and weight bearing activities [5]. EMG is a strategy used to assess and record the electrical activity created by skeletal muscles. EMG is performed utilizing an instrument called an electromyography to deliver a record called an EMG. Electromyography identifies the electrical potential produced by muscle cells when such cells are electrically or neurologically actuate.

Numerous regular therapeutic conditions, for example, diabetes, can bring about nerve harm. In such cases, the longest nerves are typically influenced to start with, subsequently the term fringe neuropathy. Notwithstanding when diabetes is controlled with eating regimen or oral and injectable drugs, nerve harm frequently happens. An EMG can assess the seriousness and screen any movement of a fringe neuropathy. Myopathy is an ailment that is restricted to the muscle and muscle-supporting structures. Myopathies are innate (acquired from a mother or father) or procured (from contamination or hidden medicinal conditions). A patient will typically give proximal muscle shortcoming and maybe myalgia (muscle throbs). An EMG can limit the sickness process in such cases to help analysis [3]. Central neuropathies happen when a solitary nerve endures harm at a particular site along its course. A boundless number of conceivable central neuropathies exist in the body, yet the most widely recognized case is carpal passage disorder. Carpal passage disorder happens when the nerve to the hand is pressed at the wrist, bringing about deadness, shivering, and torment. An EMG assesses the seriousness and area of such central neuropathies.

In this work, we have considered EMG data which is acquired from the skeletal muscles which are responsible for knee joint flexion and extension movements. The EMG data is acquired during different posture and activities, such as standing, sitting, standing to sitting, sitting to standing and during walking. For diagnosis of diabetes we propose a novel hybrid model based on machine learning method. Unsupervised and supervised learning algorithms are used for training the model and model building, respectively, which are based on wavelet features. Furthermore, the performance of the proposed model is given in terms of prediction of diabetes with high accuracy, sensitivity, and specificity, which outperforms other techniques. 
Anjaneya L.H., Mallikarjun S. Holi and Chandrashekar S.; A Machine Learning Technique to Analyze Surface EMG Signals in Normal and Diabetic Subjects. Journal of Biomedical Engineering and Medical Imaging, Volume 3, No 3, June (2016), pp 1-14

The paper is organized as follows. A brief literature survey on diabetes classification is provided in Section II. The proposed methodology is presented in Section III, followed by results and discussion in Section IV and conclusions is provided in Section V.

\section{Literature Survey}

Diabetes mellitus is an incessant illness broadly utilized as a part of the populace and consistently expanding. The long term complications are numerous and refuting, among these the diabetic foot, floated from the contemporary vicinity of fringe neuropathy and fringe blood vessel sickness, that changing the biomechanics of the foot, can convey to callosity arrangement and ulcerations. Diabetic neuropathy is the most widely recognized endless entanglement connected with diabetes mellitus, influencing $20-50 \%$ of diabetic patients 10 years after their finding and prompts a dynamic loss of somatosensory affectability, proprioception and distal muscle work particularly in the lower extremities, which may bring about an adjustment of the control during standing and gait [6]. Fringe neuropathy and fringe blood vessel sickness are the most widely recognized and nullifying diabetes' inconveniences, included in the pathogenesis of diabetic foot. They represent the main source of non-traumatic lower appendage removals. It results from two components. The first is a decreased blow of blood in the subpar appendages, brought on from the vicinity of crushing fringe arteriopathy ailment. Peripheral arteriopathy disease is a perpetual obstructive illness of the supply routes of the lower appendage brought about by atherosclerosis. The lessening in blood stream can bring about side effects of torment in the lower appendage on activity, known as discontinuous claudication. Together with diabetes falls in more seasoned grown-ups are a noteworthy general wellbeing concern and have given a great part of the inspiration to explore into age-related changes in human step. Stumbling amid strolling is the dominating reason for falls in the elderly as well as in the neuropathic subjects.

The social and financial weight of the diabetic foot can be lessened through a brief conclusion and treatment from the earliest starting point. Diabetic foot issues are identified with, fringe neuropathy, foot injury, foot deformation, expanded foot weights, and callus [7]. Mortality and dreariness identified with ulceration is still high and recuperated ulcers frequently repeat [8]. Distal symmetric sensorimotor polyneuropathy, is principally kept to the axons of little and vast fiber tangible afferents. The outcome is a "loading feet" example of tangible misfortune that starts in the toes and advances proximally [9]. Fringe neuropathy patients display diminished steadiness while standing [5-7] and in addition amid element conditions. Researchers $[10,11]$ discovered step design deviations in diabetic patients with fringe neuropathy (DPN) and without fringe neuropathy (NoDPN). Case in point imperative deviations were uncovered in hip, knee, lower leg joints and trunk minute examples over the whole position period of step in both DPN and NoDPN subjects [10]. These discoveries set up the requirement for researching the part of muscles actuation in diabetic stride variations from the norm notwithstanding when neuropathy is not present. A few researchers $[11,12]$ reported variations from the norm in the sEMG example of vastus lateralis and tibialis foremost, soleus, average and sidelong gastrocnemius and, average hamstrings of DPN subjects. Wells (1981) [13] found that the ground response strategy is a decent indicator of net joint moments for moderate step, however expanding the speed of stride results in expanded errors, particularly at the hip. Hence for healthy populaces, the connected portion system is desirable over compute joint moments; the connected section strategy looks into the mass-quickening results of the foot, leg and thigh that the ground response technique dismisses. Motkaet al. [14] analyzed four different approaches for the classification of subjects into two classes namely: Diabetic \& 
Non-diabetic. The techniques undertaken are ANFIS, PCA + ANFIS, Neural Networks and PCA + Neural Networks.

\section{Proposed Methodology}

\subsection{Data Collection}

In the experimental study for the proposed system we have collected lower limb surface EMG (sEMG) using electromyography system (RMS EMG-EP MK-11 Version 1.1 from Recorders and Medicare Systems). This dataset contains SEMG data of 22 subjects, out of 22, 11 subjects suffer from knee abnormality with diabetes and remaining 11 with knee abnormality without diabetes. Each subject has 3 set of data recording, one sitting, one standing and one gait. In this experiment 4 electrodes have been used to record the data which are corresponding to the time series for each channel. Each series contains $\sim 5$ shares or motion repetitions for each subject.

All subjects are asked to perform three activities to analyze the behavior of knee muscle, leg flexion and extension in sitting position, standing and gait. The surface EMG signals are acquired with the help of 4 electrodes (i.e. 4-channels, Ch1 to Ch4) which are placed on the appropriate locations on the surface of the body to acquire the signals from vastusmedialis (VM, standing), semitendinosus (ST, sitting), biceps femoris (BF, knee movement) and rectus femoris (RF, gait), and channel-5 (Ch5) is used for measurement of knee flexion angle (FX) using goniometer, the details are as shown in Table 1. This dataset contains 10 attributes as shown in Table 2.

Table1. Details of sEMG Data from Different Muscles and Knee Flexion Angle.

\begin{tabular}{|c|c|c|c|c|c|}
\hline Segment & \multicolumn{4}{|c|}{ sEMG } & $\begin{array}{c}\text { Knee Flexion } \\
\text { Angle }\end{array}$ \\
\hline Channel & Ch1 & Ch2 & Ch3 & Ch4 & Ch5 \\
\hline Muscle & RF & BF & VM & ST & FX \\
\hline
\end{tabular}

Table2. Attributes and Labelling

\begin{tabular}{|c|c|}
\hline Dataset Attributes & Labeling of Data \\
\hline Age & Age \\
\hline Abnormal & $\mathrm{A}$ \\
\hline Marching / Gait & Mar \\
\hline Semitendinosus & $\mathrm{ST}$ \\
\hline Normal & $\mathrm{N}$ \\
\hline Rectus Femoris & RF \\
\hline Biceps Femoris & $\mathrm{BF}$ \\
\hline Class & Class \\
\hline Vastus Medialis & VM \\
\hline Flexor-Extension Angle & FM \\
\hline
\end{tabular}

In the given dataset class value varies 0 or 1 . Class 1 is represented as tested positive for diabetes and class 0 presents the tested negative for diabetes, i.e. normal. Total number of instance with respect to their classes are given below

\begin{tabular}{|c|c|}
\hline Class & Instances \\
\hline 0 & 11 \\
\hline 1 & 11 \\
\hline
\end{tabular}


Anjaneya L.H., Mallikarjun S. Holi and Chandrashekar S.; A Machine Learning Technique to Analyze Surface EMG Signals in Normal and Diabetic Subjects. Journal of Biomedical Engineering and Medical Imaging, Volume 3, No 3, June (2016), pp 1-14

The Figure 1 shows the block diagram typical sEMG data acquisition system which is used in the present study.

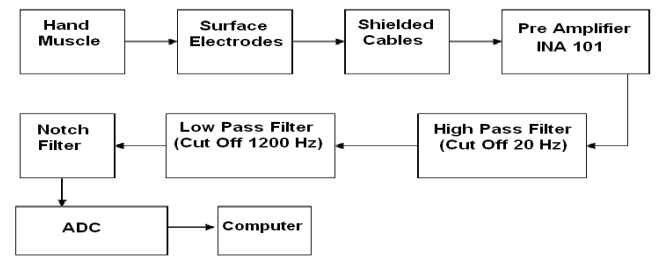

Figure 1. Block diagram of sEMG data acquisition system

The raw SEMG signals are acquired by placing the body surface electrodes on a region of interest, which reflects the electrical activity of the muscle fibers active at that position and time. The sensed EMG signals are processed through the stages like amplification, filtration and fed to the computer through the ADC for the analysis purpose. The acquired EMG signals are amplified since the obtained signals are in the range of few microvolts, then they are processed through a high pass and low pass filters with a range of $20 \mathrm{~Hz}$ to $1200 \mathrm{~Hz}$. The filtered signals are fed to the notch filter with $50 \mathrm{~Hz}$ cutoff to eliminate the power line distortions that exists along with the EMG signals. Waveforms are often sampled faster than the Nyquist rate to obtain desired temporal resolution, even though, theoretically, oversampling adds no information and should not be necessary.

\subsection{Wavelet feature Extraction}

Wavelet analysis can be done by two methods; discrete wavelet transform (DWT) and continuous wavelet transform (CWT). DWT was chosen in this study as a result of the focus progressively building applications. DWT is a strategy that iteratively changes an intrigued sign into multi-determination subsets of coefficients. Like the ordinary time-frequency investigation, the DWT changes the EMG signal with a suitable wavelet basis function (WF). Along these lines, the WF assumes a key part in the multidetermination examination. In this study, we researched the helpfulness of the multi-determination examination by concentrating on the EMG features with diverse scales and nearby varieties, furthermore the disposal of the undesired frequency segments. Additionally, the choice of an ideal WF is proposed [19].

The first EMG signal (S) is passed through a low-pass and high-pass filters (coefficients of filters rely upon WF) to get an estimate coefficient subset (cA1) and a subtle element coefficient subset (cD1) at the first level. Keeping in mind the end goal to acquire the various determination subsets, redundant change is finished. This procedure is repeated until the desired last level is acquired. In this study, four levels of disintegration are chosen. In the EMG investigation, four levels of wavelet disintegration show preferable execution over alternate levels in a great deal of literary works [14]. This process creates the coefficient subsets of the level 4 estimate (cA4) and the level 1, 2, 3, 4 subtle elements (cD1, cD2, cD3, and $(\mathrm{D} 4$ ), individually. Also, every coefficient subset can be recreated to acquire a successful EMG flag part. Using so as to remake of a sign is finished the converse wavelet change. For the most part, the converse change is performed by utilizing the coefficients of the considerable number of segments of the last level disintegration, which is the fourth level close estimation and the initial four levels of subtle element ( $C A 4, C D 1, C D 2, c D 3$, and $C D 4)$. On the other hand, in this study, we characterize the reconstruction EMG signal by the reversal of subset reliance. For instance, with a specific end goal to 
acquire the evaluated sign from close estimation coefficient subset, the reproduced EMG signal (A4) is inversed by utilizing the fourth level coefficients (cA4). Along these lines, we will acquire the reconstructed EMG signals, to be specific A4, D4, D3, D2, and D1 that are reproduced from CA4, CD4, $c D 3, c D 2$, and $c D 1$, individually. Be that as it may, the ideal wavelet capacity is reliant on the kind of intrigued applications seven mother wavelets are chosen to be assessed in this study. There are the second and the seventh requests of Daubechies wavelet ( $d b 2$ and $d b 7$ ), the forward and the fifth requests of Coiflet wavelet (coif4 and coif5), the fifth request of Symlets wavelet (sym5), the fifth request of BioSplines wavelet (bior5.5), and the second request of ReverseBior wavelet (rbio2.2). After that, the wavelet coefficient subsets (cD1-cD4, cA4) and the reconstructed EMG signals (D1-D4, A4) were extricated from their elements. The Fig. 2 shows the procedure of sEMG feature extraction by wavelet decomposition [20]

In assessing execution of the EMG components, class distinctness perspective is a focal measure. The great quality in class detachability perspective implies that the consequence of misclassification will be as low as could be expected under the circumstances. In this study, we utilized two assessment criteria, called the dissipate diagram and the factual computation. For the most part, the choice of EMG components can be conveyed in view of either classifier technique or factual estimation list. Then again, a downside of an assessment utilizing classifier is that the assessment results are subject to sorts of the classifier. Consequently, in this study, we have proposed the choice of EMG elements in view of measurable list.

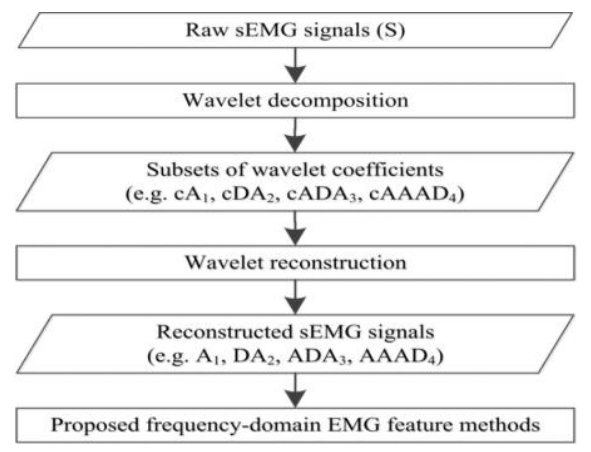

Figure 2. Procedure of the extraction of sEMG features by wavelet decomposition.

The Figure 2 shows the feature extraction stage using wavelet methodology. Initially raw EMG signal is processed and given to the wavelet decomposition stage which results in the decomposition of the given signal. During next stage coefficients are computed and wavelet reconstruction takes place to achieve the frequency-domain reconstructed signal. Finally the features of this signal are extracted.

The Fig. 3 presents the overall architecture of the proposed system. First of all the EMG data is collected and passed through the preprocessing step to remove the noise. The next step is the extraction of wavelet features and finally based on these wavelet features neural network is trained to achieve the classification of subjects as normal and diabetic. 


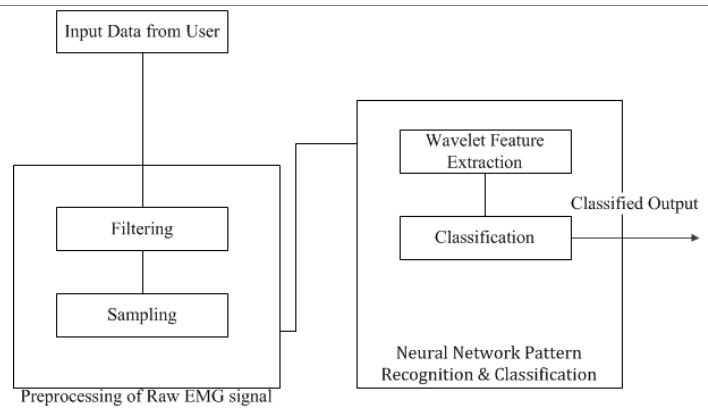

Figure 3. Schematic diagram of the proposed work

The Figure 4 presents the feature extraction process for the EMG signal. In this step 4 level decomposition coefficients are extracted using wavelet decomposition. First of all, original signal is decomposed then coefficients are extracted as features of the signal. These coefficients can be calculated by using equation (1) and equation (2).

Wavelet transform is the convolution of $\alpha(t)$ with a signalS $(t)$. These discrete wavelets are associated with the scale function $\gamma(t)$. By performing convolution on the signal approximation coefficients $C$ can be calculated. DWT can be presented as

$$
\mathcal{T}_{m, n}=\int_{-\infty}^{\infty} S(t) \alpha_{m, n}(t) d t
$$

The approximation coefficient of the signal at the scale $\mathrm{m}$ and location $\mathrm{n}$ can be written as

$$
\mathcal{C}_{m, n}=\int_{-\infty}^{\infty} S(t) \gamma_{m, n}(t) d t
$$

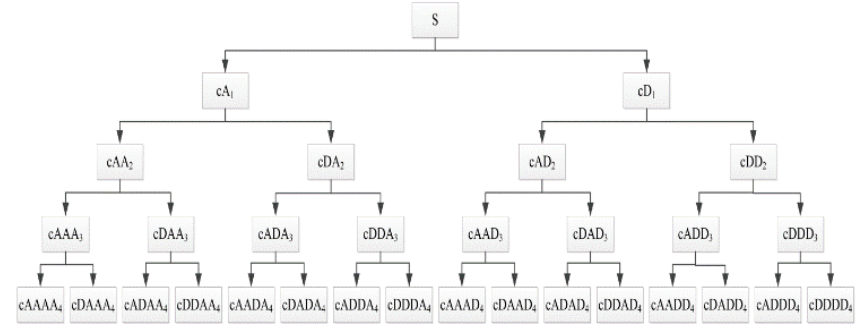

Figure 4. Wavelet feature extraction for 4- level decomposition

The Fig. 5 and Fig. 6 show the decomposed signal after the application of DWT on the sEMG of diabetic and normal subjects. It can be observed that there is a significant difference in the decomposed signal and wavelet coefficients of normal and diabetic subjects. Hence these coefficients can be used as features to classify the data as normal and diabetic based on the SEMG signal. The data was analyzed for all the 5 set of sEMG data acquired during different physical activities for normal and diabetic subjects. The wavelet coefficients were extracted for the data, the mean and standard deviation were calculated 
as shown in Table 3. The data was analyzed statistically by student's $t$-test at $95 \%$ confidence level. It is found the mean values of the normal and diabetic subjects is found to be significantly different $(p<0.05)$.

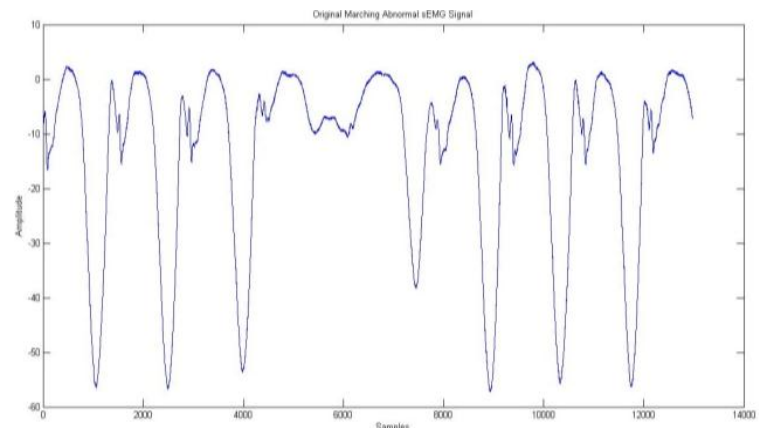

(a) Original Signal

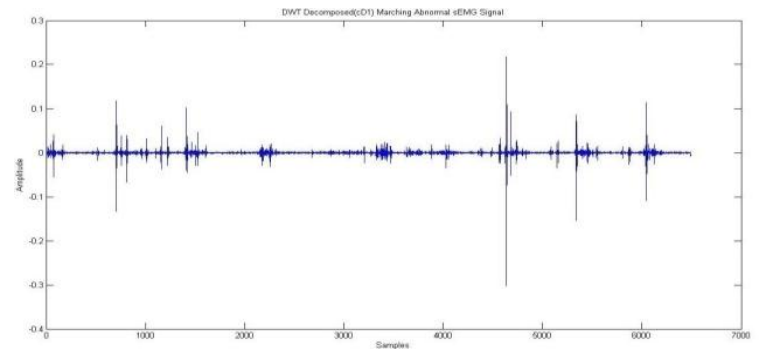

(c) First order wavelet coefficient

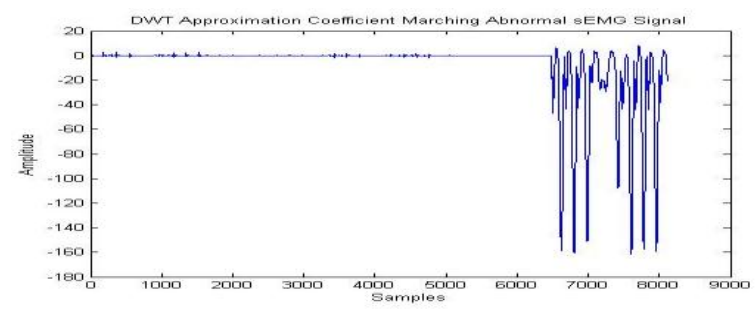

(e ) Approximation wavelet Coefficient

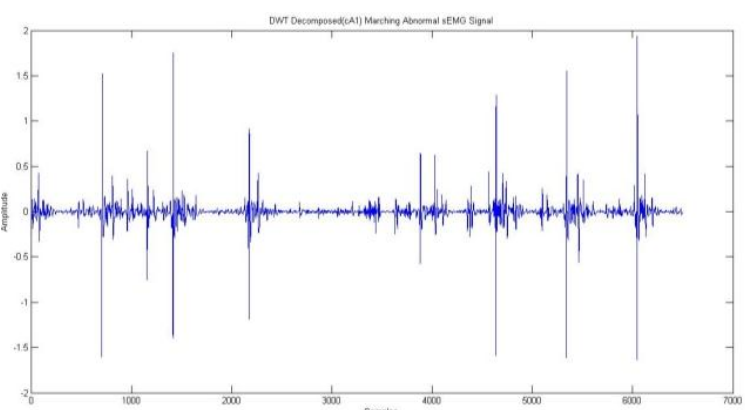

(b) Wavelet Decomposed Signal

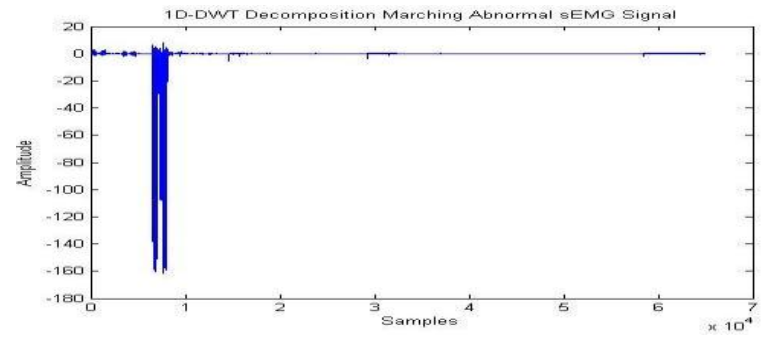

(d) $2^{\text {nd }}$ Order wavelet coefficient

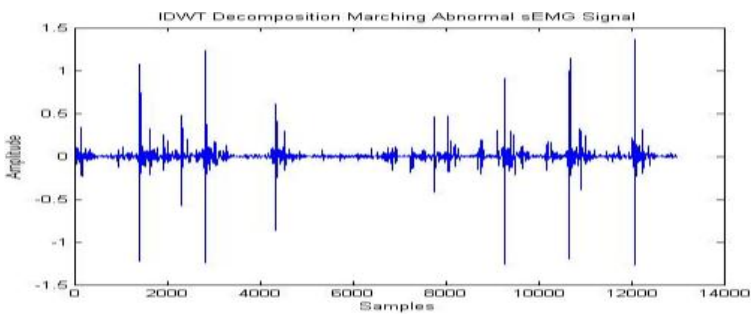

(f) IDWT decomposition

Figure 5. Wavelet coefficients for DWT feature extraction from SEMG of diabetic subject

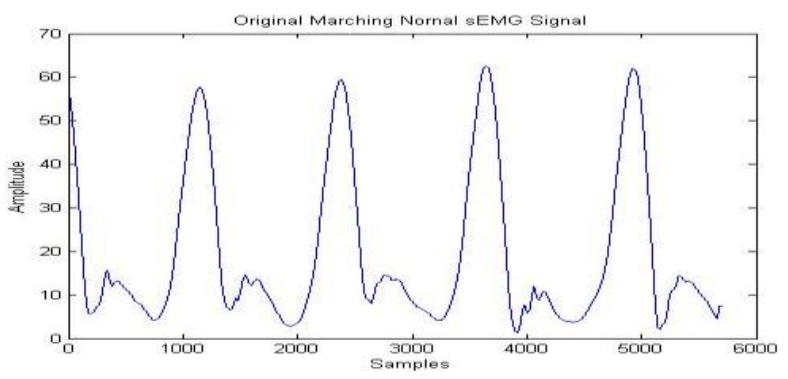

(a) Original Signal

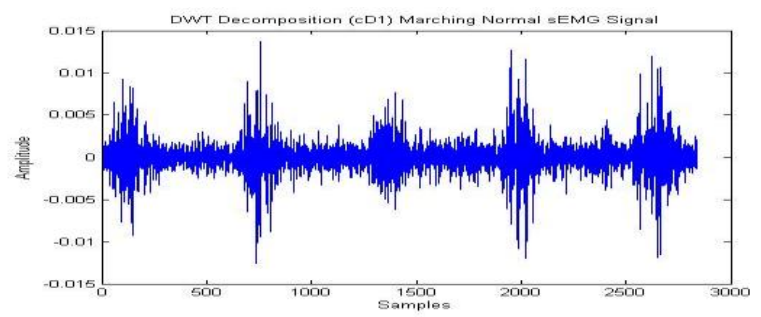

(c) First order wavelet coefficient

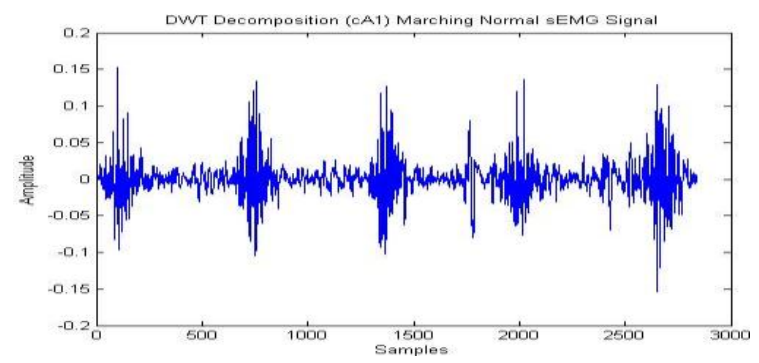

(b) Wavelet Decomposed Signal

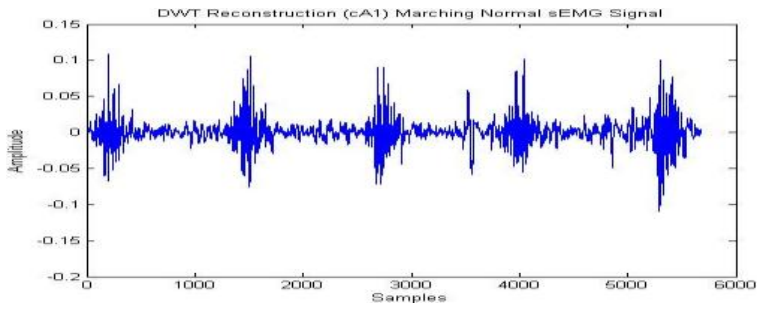

(d) 2nd Order wavelet coefficient 
Anjaneya L.H., Mallikarjun S. Holi and Chandrashekar S.; A Machine Learning Technique to Analyze Surface EMG Signals in Normal and Diabetic Subjects. Journal of Biomedical Engineering and Medical Imaging, Volume 3, No 3, June (2016), pp 1-14

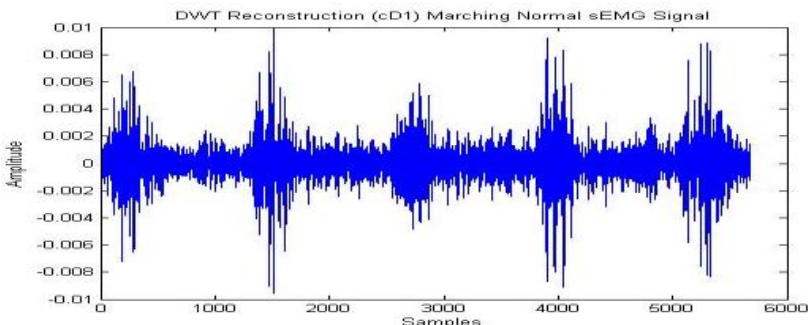

(e ) cD1wavelet Coefficient

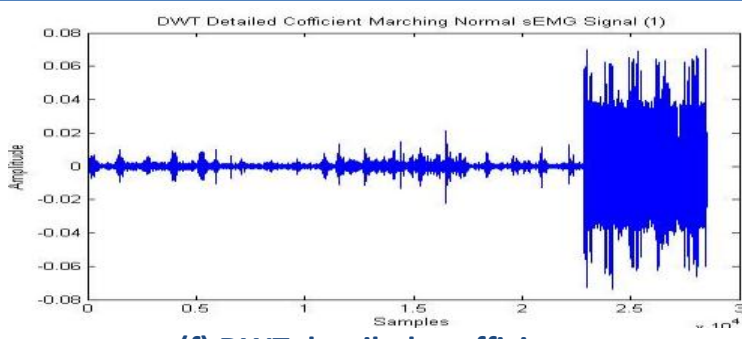

(f) DWT detailed coefficients

Figure 6. Wavelet coefficients for DWT feature extraction from normal subject

Table 3. Data Statistics for Normal and Abnormal Subjects

\begin{tabular}{|c|c|c|c|c|}
\hline $\begin{array}{l}\text { Physical } \\
\text { Activity }\end{array}$ & $\begin{array}{l}\text { Subject Type } \\
\text { (Normal / } \\
\text { Abnormal) }\end{array}$ & Mean & $\begin{array}{l}\text { Standard } \\
\text { Deviation }\end{array}$ & $\begin{array}{c}\text { Statistical } \\
\text { significance }\end{array}$ \\
\hline \multirow{2}{*}{$\begin{array}{l}\text { Marching } \\
\text { (Gait) }\end{array}$} & Abnormal & $3.1296 \mathrm{e}-04$ & 0.0023 & \multirow{2}{*}{$p<0.05$} \\
\hline & Normal & $9.3887 \mathrm{e}-04$ & 0.0039 & \\
\hline \multirow{2}{*}{$\begin{array}{c}\text { Leg } \\
\text { Extension } \\
\text { (sitting) }\end{array}$} & Abnormal & $2.9178 \mathrm{e}-04$ & 0.0020 & \multirow[b]{2}{*}{$p<0.05$} \\
\hline & Normal & $2.4985 \mathrm{e}-04$ & $2.7173 e-04$ & \\
\hline \multirow{2}{*}{ Stand up } & Abnormal & 0.0040 & 0.0134 & \multirow{2}{*}{$p<0.05$} \\
\hline & Normal & 0.0191 & 0.0246 & \\
\hline
\end{tabular}

\subsection{Wavelet feature Extraction}

Neural systems are prescient model that have capacity to learn, dissect, compose the information and foresee test outcomes in like manner. Among a few sorts of neural systems, food forward neural system is generally utilized in medicinal finding applications and others. These systems are prepared by an arrangement of examples called preparing set, whose result is now known. In our study, multilayer perceptron feed forward back proliferation neural network prepared with Levenberg Marquardt (LM) calculation is connected for classification. LM preparing algorithm performs efficiently minima and produces a superior expense capacity [12]. Feed forward NN comprises of information, shrouded and a yield layer, and the information works in forward course, and the mistake is back spread to overhaul the weights at each age with a specific end goal to diminish lapses. Table 4 gives the configuration of ANN model, and Fig. 7 shows the general architecture of ANN.

Table 4. Configuration of ANN Model

\begin{tabular}{|c|c|}
\hline ANN Parameter & Wavelet coefficients \\
\hline Hidden Layer & 5 \\
\hline Learning Rate & $50,75,100$ (in $\%)$ \\
\hline Validation Ratio & $50,75,100$ (in \%) \\
\hline Training Rate & $50,75,100$ (in \%) \\
\hline Number of Epochs & 1000 \\
\hline Target Class & 2 \\
\hline
\end{tabular}

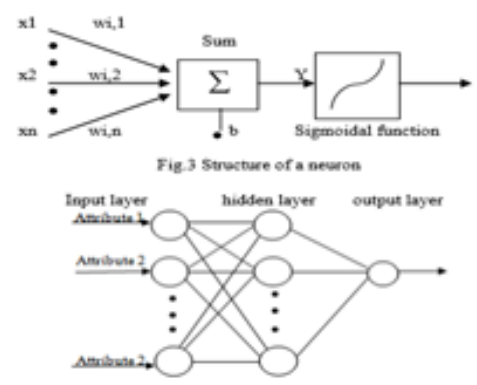

Figure 7. Architecture of neural network 
Working of neural network is given below:

- Randomly choose the initial weights, while error is too large

For each training pattern (presented in random order)

- Apply the inputs to the network

- Calculate the output for every neuron through the hidden layer(s), to the output layer.

- $\quad$ Calculate the error at the outputs.

- Use the output error to compute error signals for pre-output layers.

- $\quad$ Use the error signals to compute weight adjustments.

- Apply the weight adjustments.

- Periodically evaluate the network performance.

\section{Results and Discussions}

This section presents the diabetes classification results by using artificial neural network. Extracted features contain most of the features based on the time domain and some from the frequency domain that are calculated based on amplitude and energy properties. In this group, the resultant indices calculated from the second-level reconstructed SEMG signal (D2) with Db7 showed better class separability in feature space compared to the RES indices calculated from the original sEMG signal (S).

Table 5. Classification Results For Diabetic and Non-Diabetic Data Using Artificial Neural Network

\begin{tabular}{|c|c|c|c|c|c|}
\hline $\begin{array}{c}\text { Learning } \\
\text { Rate }\end{array}$ & $\begin{array}{c}\text { Train } \\
\text { Ratio }\end{array}$ & $\begin{array}{c}\text { Validation } \\
\text { ratio }\end{array}$ & $\begin{array}{c}\text { Test } \\
\text { Ratio }\end{array}$ & Error & Accuracy \\
\hline 100 & 7 & 5 & 6 & $1 \mathrm{e}-0100$ & 95.45 \\
\hline 100 & 7 & 0 & 6 & 1.1000 & 90.90 \\
\hline 90 & 6 & 4 & 5 & 1.2 & 81.81 \\
\hline
\end{tabular}

Learning rate, train ratio, validation ratio, test ratio and error are the user defined input for the neural network, based on these parameters training, testing and validation takes place to achieve the classification accuracy.

Above table 4 shows the classification results for varied parameters. Neural network performance depends upon the learning rate, training ratio, testing ratio. In our framework initially we trained our network with train ratio 7, learning ratio 100, validation ratio 5 and test ratio is 6 . By using this configuration we achieve the accuracy of $95.45 \%$. In second case learning rate we keep same as previous, train ratio 1 , validation ratio 1 and test ratio 1 . This configuration gives the accuracy of $90.90 \%$. In $3^{\text {rd }}$ case the learning ratio is fixed at 90 , training ratio 6 , validation ratio 4 and testing ratio is 5 . By this we achieve the classification accuracy 81.81 .

Table 6. Precision Vs Recall

\begin{tabular}{|c|c|}
\hline PRECISION & RECALL \\
\hline 1 & 1 \\
\hline 1 & 0.989796 \\
\hline
\end{tabular}




\section{Table 7. Statistical Results}

Case-1

\begin{tabular}{|l|l|}
\hline TP (True Positive) & 20 \\
\hline TN (True Negative) & 1 \\
\hline
\end{tabular}

Case-2

\begin{tabular}{|l|l|}
\hline TP (True Positive) & 18 \\
\hline TN (True Negative) & 2 \\
\hline
\end{tabular}

Case-3

\begin{tabular}{|l|l|}
\hline TP (True Positive) & 16 \\
\hline TN (True Negative) & 2 \\
\hline
\end{tabular}

\begin{tabular}{|c|c|c|}
\hline & $\begin{array}{c}\text { Prediction of Class } \\
\text { (Positive) }\end{array}$ & $\begin{array}{c}\text { Prediction of Class } \\
\text { (Negative) }\end{array}$ \\
\hline Actual : Positive & TP & FN \\
\hline Actual : Negative & FP & TN \\
\hline
\end{tabular}

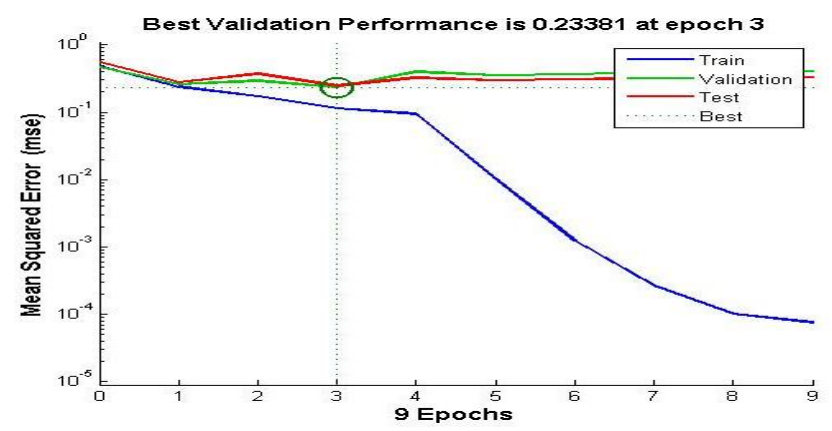

Figure 8. Validation performance of neural network

The above given figure is the validation performance of the neural network. For first case to achieve the accuracy of $95.45 \%$ it utilizes 192 epochs. This figure shows the mean square error while training the neural network.
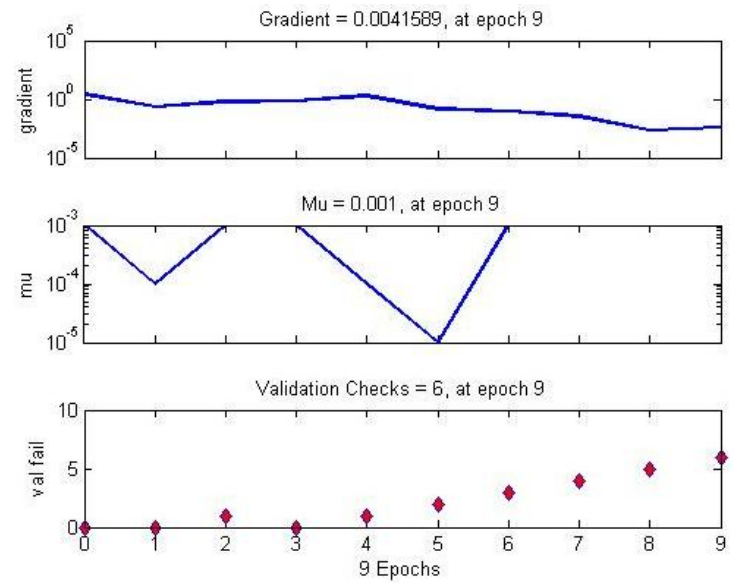

Figure 9. Validation performance of neural network 
Figure 9 represents the validation check for the neural network training. For 192 epochs it shows the validation checks. It computes the failing constraints at the training stage of neural network. Validation check performance can be measured by gradient and mu.
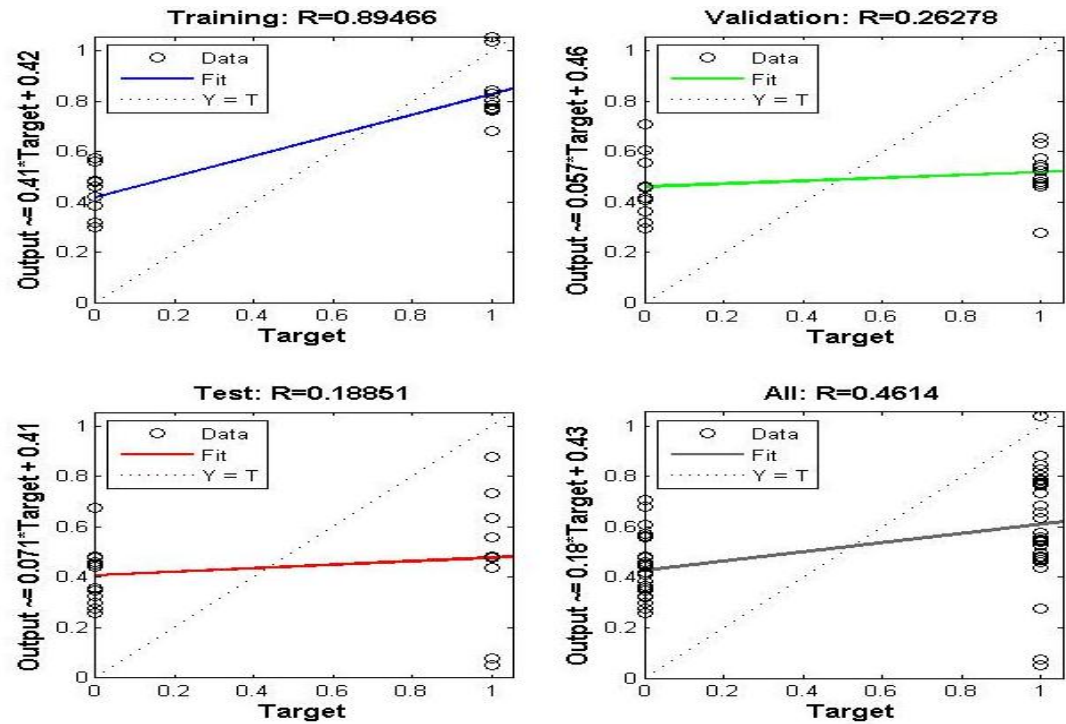

Figure 10. Training and validation performance of the proposed system

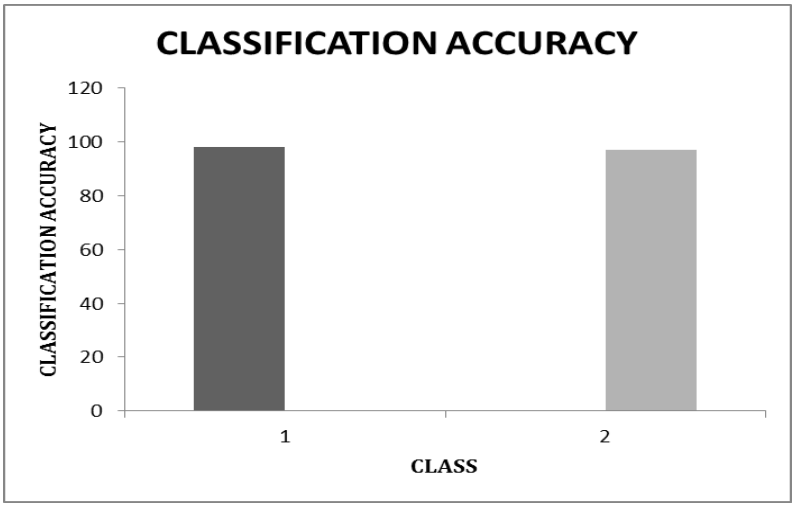

Figure 11. Classification accuracy for Diabetic and non-Diabetic data

This figure shows the classification accuracy of proposed classification scheme for diabetes. These results are achieved by using the confusion matrix. Precision (also called positive predictive value) is the fraction of retrieved instances that are relevant, while recall (also known as sensitivity) is the fraction of relevant instances that are retrieved. Both precision and recall are therefore based on an understanding and measure of relevance. Precision and recall can be calculated as

$$
\begin{gathered}
\text { Precision }=T P /(T P+F P) \\
\text { Recall }=T P /(T P+F N)
\end{gathered}
$$

- Sensitivity (also called the true positive rate or the recall in some fields) measures the proportion of positives that are correctly identified. 
Anjaneya L.H., Mallikarjun S. Holi and Chandrashekar S.; A Machine Learning Technique to Analyze Surface EMG Signals in Normal and Diabetic Subjects. Journal of Biomedical Engineering and Medical Imaging, Volume 3, No 3, June (2016), pp 1-14

- Specificity (also called the true negative rate) measures the proportion of negatives that are correctly identified.

- The main advantage of these statistics is that they are focused on the performance of the models on the events, completely ignoring their accurate predictions for the non-event classes. Informally, precision measures the proportion of events signaled by the model that are real events.

- Recall measures the proportion of events occurring in the domain that are "captured" by the models.

\section{Conclusion}

The ANN classifier was used to classify two classes of EMG signals when the combined features of wavelet coefficients of the EMG signals and AR modeling were used as inputs. In this work wavelet transform based approach is used for the feature extraction and to classify the diabetes artificial neural networks is utilized. The ANN techniques are capable of classifying the EMG signals with a high degree of accuracy and repeatability. The total classification accuracy is also obtained with $95.45 \%$. This demonstrates that the ANN classifier can be valuable for the capture and expression of knowledge useful to a clinician. These results provide encouragement to develop and evaluate an ANN method for quantifying the level of contribution of classifying the diabetes and non-diabetes data.

\section{REFERENCES}

[1] Castilho, L.V.; Lopes, H.S.; Tacla, C.A., "Modeling and Building an Ontology for Neuropediatric Physiotherapy Domain", Eighth International Conference on Hybrid Intelligent Systems, HIS '08, vol., no., pp.210-215, 10-12 Sept. 2008

[2] American Diabetes Association. "Diagnosis and Classification of Diabetes Mellitus", Diabetes Care 36.Suppl 1 (2013): S67-S74. PMC.Web. 25 Sept. 2015.

[3] International Diabetes Federation, Diabetes Atlas, 3rd ed. Brussels, Belgium: International Diabetes Federation, 2007.

[4] Barakat, N.; Bradley, A.P.; Barakat, M.N.H., "Intelligible Support Vector Machines for Diagnosis of Diabetes Mellitus", IEEE Transactions on Information Technology in Biomedicine, vol. 14, no.4, pp. 1114-1120, July 2010

[5] M. Z. Al-Faiz, Yousif. I. Al-Mashhadany, "Human Arm Movements Recognition Based on EMG Signal", MASAUM Journal Of Basic and Applied Sciences (MJBAS), vol. 1, issue 2, pp. 164-171, September 2009.

[6] Sacco ICN, Amadio AC. "Influence of the diabetic neuropathy on the behavior of electromyographic and sensorial responses in treadmill gait", Clinical Biomechanics 2003; 18426-434, 2003.

[7] Van Schie, $\mathrm{CH}$, Rawat F, Boulton, AJ. "Reduction of plantar pressure using a prototype pressurerelieving dressing", Diabetes Care, 28(9):2236-7, 2005. 
[8] Lawall H, Diehm C., "Diabetic foot syndrome from the perspective of angiology and diabetology", 38(12):1149-59, 2009.

[9] Greene DA, Sima AA, Stevens MJ, Feldman EL, Lattimer SA, "Complications: neuropathy, pathogenetic considerations", Diabetes Care, 15(12):1902-25, 1992.

[10] Katoulis EC, Ebdon-Parry M, Lanshammar H, Vileikyte L, Kulkarni J, Boulton AJ, “Gait abnormalities in diabetic neuropathy", 20(12):1904-7, 1997.

[11] Sawacha Z, Gabriella G, Cristoferi G, Guiotto A, Avogaro A, Cobelli C., "Diabetic gait and posture abnormalities: a biomechanical investigation through three dimensional gait analysis", Clin Biomech, 24(9):722-8, 2009.

[12] Akashi PM, Sacco IC, Watari R, Hennig E., "The effect of diabetic neuropathy and previous foot ulceration in EMG and ground reaction forces during gait", Clinical Biomechanics; 23, pp. 584-592, 2008.

[13] Sawacha Z, Spolaor F, Guarneri G, "Abnormal muscle activation during gait in diabetes patients with and without neuropathy”, Gait Posture, 35(1): pp. 101-105, 2012.

[14] Motka, R.; Parmarl, V.; Kumar, B.; Verma, A.R., "Diabetes mellitus forecast using different data mining techniques," 4th International Conference on Computer and Communication Technology (ICCCT), ISBN: 978-1-4799-1571-2, pp. 99-103, 20-22 Sept. 2013.

[15] B. C. Callaghan, J. Hur, and E. L. Feldman, "Diabetic neuropathy: one disease or two?" Current Opinion in Neurology, vol. 25, pp. 536-541, 2012.

[16] J. C. Arezzo, "Clinical features and treatments of diabetic neuropathy. Quantitative sensory testing," in Textbook of Diabetic Neuropathy, A. F. Gries, N. E. Cameron, P. A. Low, and D. Ziegler, Eds., Thieme Publishing Group, Stuttgart, Germany, pp. 184-189, 2003.

[17] Mohan, V.; Pradeepa, R., "Telemedicine in Diabetes Care: In rural India, a new prevention project seeks to fill in the screening gap.," in Pulse, IEEE , vol.5, no.3, pp.22-25, May-June 2014.

[18] Kumari, Sonu; Singh, Archana, "A data mining approach for the diagnosis of diabetes mellitus," $7^{\text {th }}$ International Conference on Intelligent Systems and Control (ISCO), pp.373-375, 4-5 Jan. 2013.

[19] K. Mahaphonchaikul, D. Sueaseenak, C. Pintavirooj, M. Sangworasil and S. Tungjitkusolmun, "EMG signal feature extraction based on wavelet transform," International Conference on Electrical Engineering/Electronics Computer Telecommunications and Information Technology (ECTI-CON), Chiang Mai, pp. 327-331, 2010.

[20] G. Wang, Z. Wang, W. Chen, and J. Zhuang, "Classification of surface EMG signals using optimal wavelet packet method based on Davies-Bouldin criterion," Medical and Biological Engineering and Computing, vol. 44, no. 10, pp. 865-872, 2006. 